\title{
The Nutrient Formula Containing Eicosapentaenoic Acid and Docosahexaenoic Acid Benefits the Fatty Acid Status of Patients Receiving Long-Term Enteral Nutrition
}

\author{
Mitsutoshi Munakata, ${ }^{1,2}$ Masazumi Nishikawa, ${ }^{3}$ Noriko Togashi, ${ }^{4}$ Eiko Nio, ${ }^{4}$ \\ Yasuko Kobayashi, ${ }^{5}$ Kiyoshi OMura, ${ }^{5}$ Kazuhiro Haginoya, ${ }^{1}$ Soichiro Tanaka, ${ }^{1}$ \\ Takuya Abe, ${ }^{3,6}$ Takanori Hishinuma, ${ }^{6}$ Nobukazu Chida, ${ }^{4}$ Shigeru Tsuchiya $^{2}$ and \\ AKIRA ONUMA ${ }^{1}$

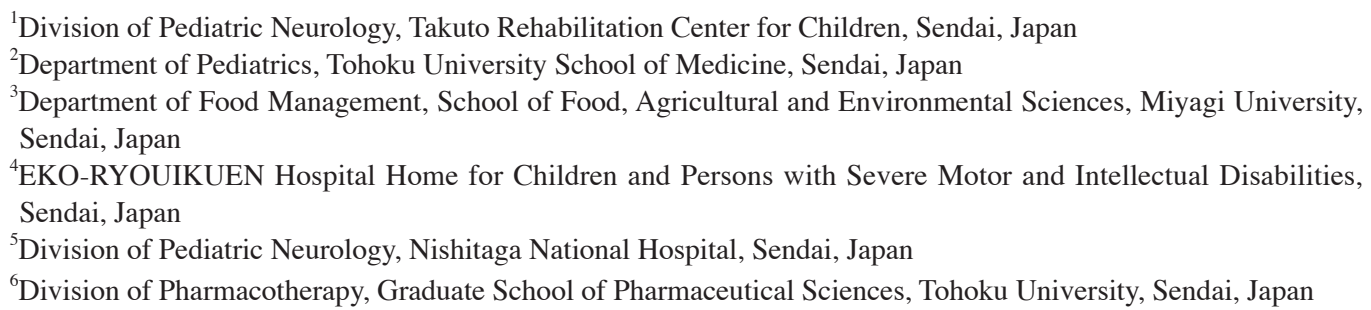

Currently, various formulas with different fatty acid compositions are used for enteral nutrition (EN). All formulas contain various concentrations of essential fatty acids: linoleic acid (LA) and $\alpha$-linolenic acid (ALA); LA is biotransformed into arachidonic acid (AA) and ALA into eicosapentaenoic acid (EPA) and docosahexaenoic acid (DHA) in vivo. Some formulas contain preformed EPA and DHA. However, the effects of the differences in the fatty acid composition on the fatty acid status of patients receiving long-term EN is not clear. We measured serum fatty acid concentrations in 50 patients with neurological diseases receiving long-term EN. The data were then compared retrospectively with reference to the fatty acid compositions of the formulas used. All of the patients received almost their entire nutritional intake via EN for at least 1 year. Blood samples were obtained just before injecting the EN solution. Among the formulas that did not include EPA or DHA, formulas with low ALA concentrations were associated with low serum EPA and DHA. Conversely, the ALA-enriched formulas with reduced LA concentrations significantly increased EPA and DHA levels, although the levels remained lower than the control values. With the formula containing EPA and DHA, the EPA and DHA levels reached control values. Therefore, the fatty acid composition of the EN formulas affected the fatty acid status of patients receiving long-term EN. Formulas containing preformed EPA and DHA with suitable amounts of essential fatty acids may benefit these patients. —— enteral nutrition; essential fatty acid; arachidonic acid; eicosapentaenoic acid; docosahexaenoic acid; dysphagia.

Tohoku J. Exp. Med., 2009, 217 (1), 23-28. (C) 2009 Tohoku University Medical Press

Patients with neurological disorders often require chronic support with enteral nutrition (EN) because of problems with eating and swallowing (Munakata 2008). For these patients, the intake of essential fatty acids depends primarily on the EN. There are two essential fatty acids for humans: linoleic acid (LA) and $\alpha$-linolenic acid (ALA) (Gerster 1995) and deficiency in these essential fatty acids can cause clinical symptoms (Richardson and Sgoutas 1975; Holman et al. 1982; Bjerve et al. 1987).

Linoleic acid is the parent of the n- 6 fatty acids, which are defined by the position of the first double bond between the 6th and 7th carbons counted from the terminal methyl group of the molecule. LA is transformed into long-chain n-6 polyunsaturated fatty acids (PUFAs), including arachidonic acid (AA). ALA is a precursor of $n-3$ fatty acids, which have the first double bond between the 3rd and 4th carbons. ALA is further transformed into long-chain $n-3$ PUFAs such as eicosapentaenoic acid (EPA) and docosahexaenoic acid (DHA); in vivo, however, the formation of EPA and DHA from ALA is limited in humans (Brenna 2002; Burdge et al. 2002). Given that the $n-6$ and $n-3$ series are not interchangeable thorough biotransformation, the systemic fatty acid status of patients receiving EN is influenced by the fatty acid composition of the EN formula (Kumode 2003).

Long-chain PUFAs such as AA, EPA, and DHA pos-

Received July 28, 2008; revision accepted for publication November 30, 2008.

Correspondence: Mitsutoshi Munakata, M.D., Ph.D., Department of Pediatrics, Tohoku University School of Medicine, Sendai 980-8574, Japan.

e-mail: m-munakata@umin.ac.jp 
sess intrinsic physiological significance. AA is metabolized into multiple bioactive eicosanoids via the AA cascade. EPA, DHA, and their metabolites competitively inhibit the overproduction of AA-related eicosanoids, thereby suppressing aberrant inflammatory reactions, blood coagulation, and the risk of ischemic heart disease (Kinsella et al. 1990; Gerster 1995; Aiko et al. 2005). In addition, highlevel accumulation of DHA in the brain plays a role in the development of learning and cognitive functions (Innis 2007). Therefore, it is important to monitor and optimize the systemic fatty acid balance in patients receiving longterm EN.

The EN formulas that are used clinically in Japan contain various amounts of LA and ALA and are characterized by a wide range of $n-6$ to $n-3$ fatty acid ratios. Most available EN formulas do not contain AA, EPA, or DHA, although some formulas include EPA and DHA. The large variation in fatty acid content among EN formulas would be expected to result in differences in the fatty acid status of patients receiving prolonged EN. This study assessed the effects of EN formulas with different fatty acid compositions on the fatty acid status of these patients.

\section{Patients And Methods}

We examined sera from 50 patients (29 males and 21 females) with neurological diseases who had been receiving EN continuously for longer than 1 year ( $>1$ year, $n=5 ;>3$ years, $n=4 ;>5$ years, $n=41$ ) in hospitals in Miyagi, Japan. The neurological diseases in these patients were cerebral palsy (spastic, $n=30$; dyskinetic, $n=1$ ), sequelae of encephalopathy or encephalitis $(n=13)$, juvenile Parkinson's disease $(n=1)$, progressive dystonia $(n=1)$, chromosome $4 \mathrm{p}$ - syndrome $(n=1)$, Tay-Sachs disease $(n=1)$, xeroderma pigmentosum $(n=1)$, and dentatorubral-pallidoluysian atrophy $(n=1)$. All patients were non-ambulatory and received almost their entire nutritional intake from artificial liquid feeding via a nasogastric tube or gastrostomy.

The patients were retrospectively divided into the following four groups based on the fatty acid composition of the EN formula that was used for each patient. The profiles of the patients in each group along with the fatty acid compositions of the respective EN formulas used are summarized in Table 1. All the EN formulas were commercially available and the compositions of the EN formulas were obtained from the manufacturers' data. For all of the EN formulas used, $1.0 \mathrm{ml}$ of EN solution contained approximately $1.0 \mathrm{kcal}$. In the high, intermediate, and low n- 6 to n-3 ratio (n-6/n-3) EN groups, patients receiving EN formulas with similar compositions were placed in one group. Therefore, two values are given for the EN formulas in these groups. In the EPA + DHA-containing EN group, patients received a single type of $\mathrm{EN}$ formula.

High n-6/n-3 EN group: In the EN formulas used in this group, the LA concentration was high and the ALA concentration was very

TABLE 1. Summary of the patient groups and the compositions of the enteral nutrition (EN) formula used in each group.

\begin{tabular}{|c|c|c|c|c|}
\hline & High n-6/n-3 & Intermediate $n-6 / n-3$ & Low n-6/n-3 & $\mathrm{EPA}+\mathrm{DHA}$-containing \\
\hline & $\begin{array}{l}\text { Receiving formula } \\
\text { with high n- } 6 / n-3 \\
\text { ratio }\end{array}$ & $\begin{array}{l}\text { Receiving formula } \\
\text { with moderate n-6/n-3 } \\
\text { ratio }\end{array}$ & $\begin{array}{l}\text { Receiving formula } \\
\text { with low n- } 6 / n-3 \\
\text { ratio }\end{array}$ & $\begin{array}{l}\text { Receiving formula } \\
\text { containing EPA and DHA } \\
\text { with low n- } 6 / n-3 \text { ratio }\end{array}$ \\
\hline \multicolumn{5}{|l|}{ Summary of patient profile } \\
\hline$n$ & 12 & 8 & 13 & 17 \\
\hline Male/female & $7 / 5$ & $4 / 4$ & $8 / 5$ & $11 / 6$ \\
\hline Age (years) & $30 \pm 11$ & $18 \pm 14$ & $22 \pm 19$ & $31 \pm 16$ \\
\hline Caloric intake (kcal/kg) & $33 \pm 8$ & $38 \pm 26$ & $39 \pm 29$ & $32 \pm 11$ \\
\hline \multicolumn{5}{|l|}{ Composition of enteral nutrition } \\
\hline$*_{n}$ & $4 / 8$ & $3 / 5$ & $7 / 6$ & 17 \\
\hline \multicolumn{5}{|c|}{ Energy composition ( $\%$ of energy) } \\
\hline Protein & $16 / 14.1$ & $12 / 19$ & $18 / 18$ & 20 \\
\hline Total fat & $28 / 31.5$ & $39 / 27$ & $20 / 23.4$ & 25 \\
\hline Carbonhydrate & $56 / 54.5$ & $49 / 54$ & $62 / 58.8$ & 55 \\
\hline \multicolumn{5}{|c|}{ Fatty acid composition (mg/100 kcal) } \\
\hline LA $(18: 2 n-6)$ & $956 / 1992.3$ & $1458 / 1533$ & $450 / 616$ & 690 \\
\hline $\operatorname{ALA}(18: 3 n-3)$ & $28 / 45.8$ & $166 / 171$ & $150 / 156$ & 154 \\
\hline $\operatorname{EPA}(20: 5 n-3)$ & - & - & - & 8 \\
\hline DHA (22:6n-3) & - & - & - & 28 \\
\hline n-6/n-3 ratio & $34 / 44$ & $8.79 / 8.96$ & $3.0 / 3.94$ & 3.6 \\
\hline
\end{tabular}

The patients receiving long-term EN were retrospectively divided into four groups based on the fatty acid composition of the EN formula used. $* n$, number of patients receiving each kind of EN formula. In the high, intermediate, and low n-6 to n-3 ratio (n-6/n-3) EN groups, patients receiving EN formulas with similar compositions were placed in one group. Therefore, two values are given for the EN formulas in these groups. In the EPA + DHA-containing EN group, patients received a single type of EN formula. The fatty acid compositions of the formulas were obtained from the manufacturers' data. Data are expressed as means \pm S.D. LA, linoleic acid; ALA, $\alpha$-linolenic acid; AA, arachidonic acid; EPA, eicosapentaenoic acid; DHA, docosahexaenoic acid. 
low, resulting in extremely high n-6 to $n-3$ fatty acid concentration ratios (n-6/n-3 ratios) of 34 or 44 . The formulas did not contain EPA or DHA.

Intermediate n-6/n-3 EN group: The EN formulas used in this group were characterized by an increased ALA concentration. The LA concentration remained high, resulting in moderately high n-6/n-3 ratios of 8.79 or 8.96. The formulas did not contain EPA or DHA.

Low n-6/n-3 EN group: For the EN formulas in this group, the ALA concentration was almost identical to that in the intermediate n-6/n-3 group, but the LA concentration was markedly reduced, giving lower $n-6 / n-3$ ratios of 3.0 or 3.94 , which is lower than the ratio (4.0) recommended as the dietary allowance for Japanese individuals (Sugano 1996). The formulas did not contain EPA or DHA.

EPA + DHA-containing EN group: Patients in this group received an EN formula containing EPA and DHA. Both LA and ALA were also included, at an n-6/n-3 ratio of 3.6.

The patients received EN three or four times a day. Blood samples were obtained just before injecting the EN solutions, after a 6- to 8 -h fast. Then, the serum was separated and stored at $-20^{\circ} \mathrm{C}$ until analysis. In a separate procedure, fasting blood samples were obtained from normal healthy volunteers who habitually consumed balanced diets ( $n=20$; mean age, $31 \pm 13$ years; 9 males, 11 females). The objectives of this study were explained to the patients' families and to the volunteers, and informed consent was obtained from the parents or guardians of all patients and from the volunteers. The study protocol was approved by the ethics committee of Takuto Rehabilitation Center for Children, Sendai, Japan.

Total lipids were extracted from serum $(0.3-0.5 \mathrm{ml})$ using a chloroform-methanol mixture (2:1 by vol), methyl behenate (Funakoshi, Tokyo, Japan) as an internal standard and butylated hydroxytoluene (Wako Pure Chemical Industries, Osaka, Japan) as an antioxidant (Folch et al. 1957; Chida et al. 1972). The total lipid extract was stored in dry chloroform before transmethylation. An aliquot of each lipid extract was dried under $\mathrm{N}_{2}$ and saponified at $95^{\circ} \mathrm{C}$ for $60 \mathrm{~min}$ in $\mathrm{KOH}-m e t h a n o l$. The fatty acids in the saponified fraction were methylated with $14 \% \mathrm{BF}_{3}$ in methanol at $95^{\circ} \mathrm{C}$ for 2 min. The methyl esters were disorbed in hexane and injected into a gas chromatograph equipped with a flame-ionization detector (model 6890N; Agilent technologies, Santa Clara, CA, USA). Separations were performed on a capillary column $(0.25 \mathrm{~mm} \times 30 \mathrm{~m}$, DB-WAX; Agilent technologies, Santa Clara, CA, USA). The column temperature was initially $150^{\circ} \mathrm{C}$ and was increased at a rate of $2^{\circ} \mathrm{C} / \mathrm{min}$ to a final column temperature of $220^{\circ} \mathrm{C}$, which was maintained for $15 \mathrm{~min}$. The injector and detector temperatures were $250^{\circ} \mathrm{C}$. Helium was used as the carrier gas, with a splitter ratio of $1: 50$ and a linear gas velocity of $30 \mathrm{~cm} / \mathrm{s}$.

All statistical analyses were performed using $R$ (R Development Core Team 2005; http://www.r-project.org). Regression lines (Figs. 1 and 2) were determined using a least squares fitting routine after logarithmic transformation of the $\mathrm{n}-6 / \mathrm{n}-3$ ratios. Pearson's correlation coefficient was calculated and tested for significance. Data for the group analyses are presented as means \pm standard deviation (Table 2). Statistical significance of differences between two groups was tested using the Mann-Whitney test. The Kruskal-Wallis test was used to test across high, intermediate, and low n-6/n-3 EN groups.

\section{Results}

The measured values for the n-3 fatty acids (ALA, EPA, and DHA) and n-6 fatty acids (LA and AA) are plotted

\section{$\underline{\mathrm{n}-3}$ series}

a

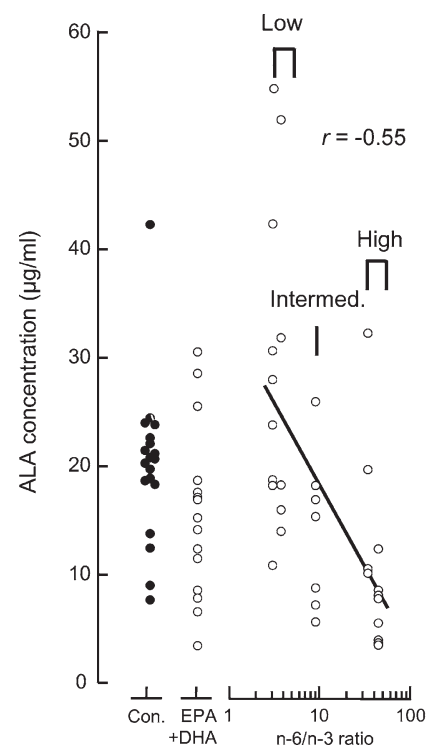

b

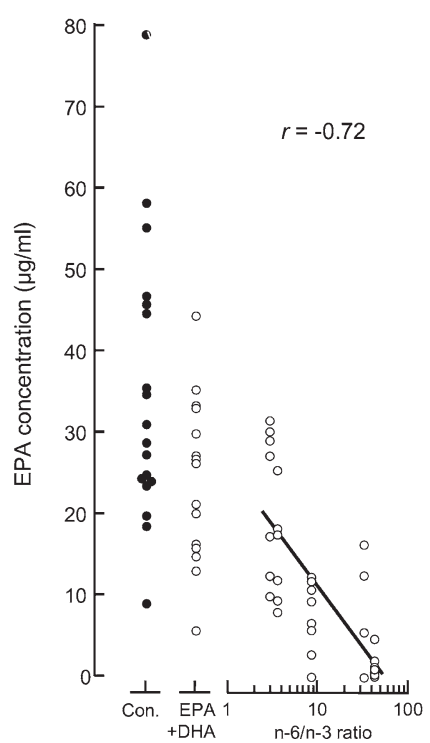

C

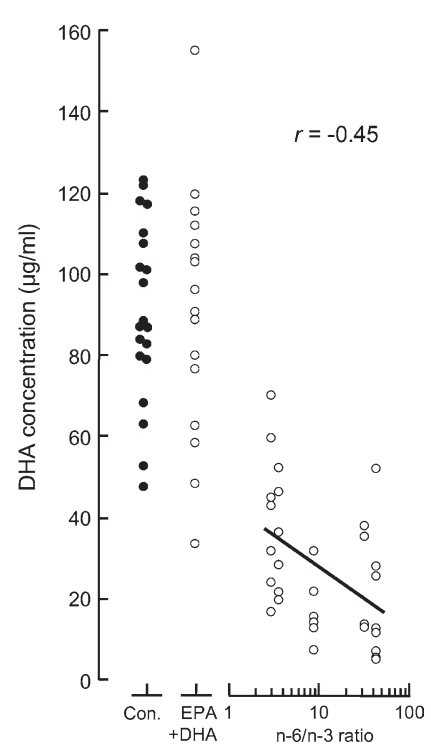

Fig. 1. Serum concentrations of n-3 fatty acids. Data from the control group (solid circles) and the EPA + DHA-containing EN group (open circles, EPA + DHA) are plotted separately. Data from the other three groups are plotted against the logarithmic axis of the n-6/n-3 ratio of the EN formula used for each patient; Low, low n-6/n-3 EN group; Intermed, intermediate n-6/n-3 EN group; High, high n-6/n-3 EN group. The solid line indicates the fit to a logarithmic function using the least squares method. $r$, correlation coefficient. 


\section{n-6 series}
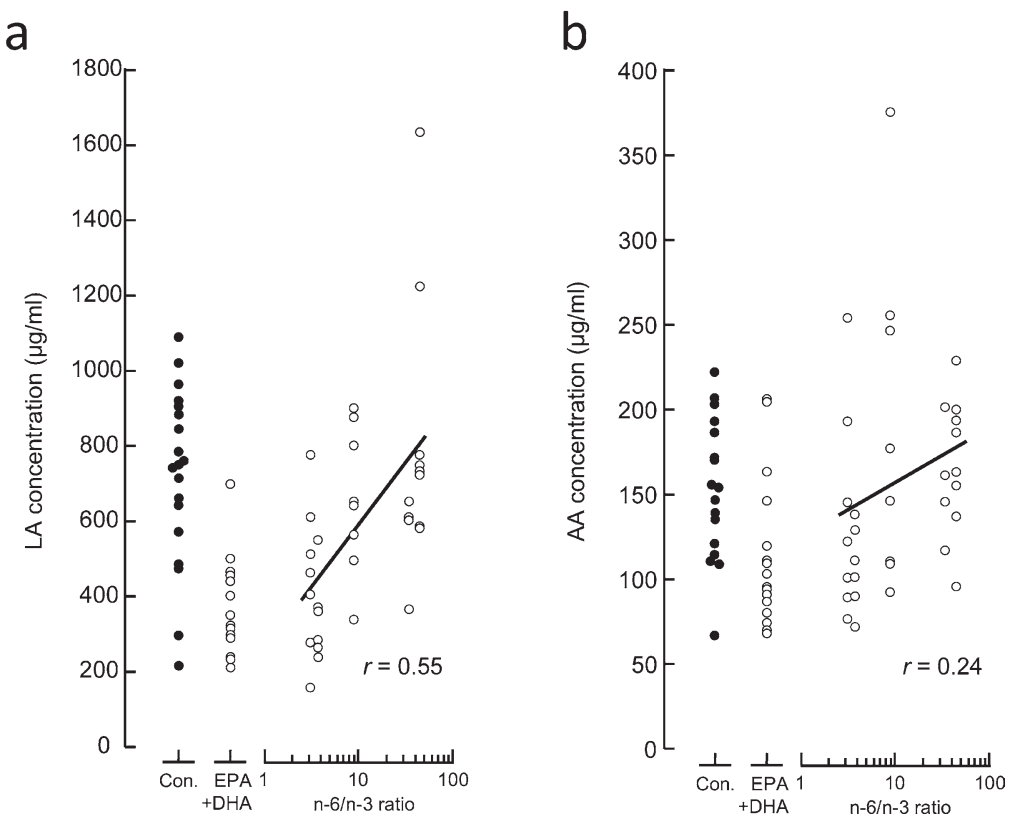

Fig. 2. Serum concentrations of n-6 fatty acids. Data from the control group (solid circles) and EPA + DHA-containing EN group (open circles, EPA + DHA) are plotted separately. Data from the low, intermediate, and high n-6/n-3 EN groups are plotted against the logarithmic axis of the $n-6 / n-3$ ratio (open circles). The solid line indicates the fit to a logarithmic function using the least squares method. $r$, correlation coefficient.

TABLE 2. Summary of the serum fatty acid concentrations.

\begin{tabular}{|c|c|c|c|c|c|c|}
\hline \multirow{2}{*}{ Group } & \multirow{2}{*}{$n$} & \multicolumn{3}{|c|}{$\mathrm{n}-3$ series $(\mu \mathrm{g} / \mathrm{ml})$} & \multicolumn{2}{|c|}{$\mathrm{n}-6$ series $(\mu \mathrm{g} / \mathrm{ml})$} \\
\hline & & ALA & EPA & DHA & LA & $\mathrm{AA}$ \\
\hline Control & 20 & $19.5 \pm 7.4$ & $35.5 \pm 17.3$ & $90.8 \pm 22.1$ & $719 \pm 230$ & $154 \pm 38$ \\
\hline High n-6/n-3 & 12 & $10.5 \pm 8.3 * *$ & $3.5 \pm 5.5 * * *$ & $21.0 \pm 15.1 * * *$ & $770 \pm 339$ & $166 \pm 39$ \\
\hline Intermediate $n-6 / n-3$ & 8 & $14.1 \pm 6.7 *$ & $7.4 \pm 4.4 * * *$ & $19.9 \pm 8.8 * * *$ & $657 \pm 193$ & $189 \pm 98$ \\
\hline Low n-6/n-3 & 13 & $26.6 \pm 14.8$ & $19.1 \pm 8.6^{* * a}$ & $38.3 \pm 16.4 * * * a$ & $406 \pm 174 * * * a$ & $125 \pm 51^{*}$ \\
\hline EPA + DHA-cintaining & 17 & $16.2 \pm 8.0$ & $24.3 \pm 10.2$ & $89.4 \pm 30.2^{\mathrm{b}}$ & $345 \pm 147 * * *$ & $113 \pm 43 * *$ \\
\hline${ }^{c} p$ value (group B-C-D) & & 0.002 & 0.0001 & 0.007 & 0.002 & 0.47 \\
\hline
\end{tabular}

Data are expressed as means \pm S.D. Significant differences between the control and the high, intermediate, low n-6/n-3 EN group, or the EPA+DHA-containing EN group (Mann-Whitney test): $* * * p<0.001$; $* * p<0.01$; and $* p<0.05$. ${ }^{*}$ Significant difference between the intermediate and low n-6/n-3 EN groups (Mann-Whitney test; $p<0.05$ ). ${ }^{\mathrm{b}}$ Significant differences between low n-6/n-3 and EPA+DHA-containing EN groups (Mann-Whitney test; $p<0.05$ ). No significant differences were detected in any fatty acid between the high and intermediate n-6/n-3 EN groups. ${ }^{c} p$-values among the low, intermediate, and high n-6/n-3 EN groups were determined using the Kruskal-Wallis test.

in Figs. 1 and 2, respectively. In the high, intermediate, and low n-6/n-3 EN groups, in which the formulas contained no EPA or DHA, the serum concentrations of ALA, EPA, and DHA increased significantly as the $n-6 / n-3$ ratio was reduced. Conversely, the LA level decreased significantly as the $n-6 / n-3$ ratio was reduced. The AA concentration tended to be low at a low $n-6 / n-3$ ratio, although this trend was not significant. In formulas containing preformed EPA and DHA, the serum EPA and DHA levels had distributions similar to those of the control values, whereas the LA and AA levels tended to be lower than the control values in the statistical analysis (Table 2).

Table 2 summarizes the values for the fatty acids and the results of the statistical analyses among the groups. In the sera from the high and intermediate n-6/n-3 EN groups, the serum levels of ALA, EPA, and DHA were significantly lower than those in the control group, whereas the LA and AA levels were not significantly different from those in the control group. The ALA and EPA levels in the intermediate n-6/n-3 EN group tended to be higher than those in the high n-6/n-3 EN group, although there were no significant differences in any of the serum fatty acids tested between these 
groups. The low n-6/n-3 EN group exhibited significantly higher serum EPA and DHA levels than those in the intermediate n-6/n-3 EN group, but the EPA and DHA levels were still significantly lower than those in the control group. Conversely, the serum concentrations of ALA, EPA, and DHA reached control values in the EPA+DHA-containing EN group. The LA and AA levels were significantly lower in the sera from the low n-6/n-3 EN and EPA + DHAcontaining EN groups, but the other groups did not show significant differences in the LA and AA levels.

ANOVA among the fatty acid levels of the high, intermediate, and low n-6/n-3 EN groups showed a significant dependency of the LA, EPA, DHA, and ALA concentrations on the $n-6 / n-3$ ratio of the EN formula; the AA concentration was an exception to this relationship.

\section{Discussion}

In patients receiving EN containing essential fatty acids but not EPA or DHA, meeting the EPA and DHA requirements relied entirely on in vivo biosynthesis from ALA. This nutritional situation resembles that of vegetarians, whose metabolism of essential fatty acids has been the focus of a number of investigations. The source of essential fatty acids for vegetarians is plant oils, most of which provide more LA than ALA with negligible amounts of longchain n-6 and n-3 PUFAs such as AA, EPA, and DHA (Davis and Kris-Etherton 2003). Owing to the low ALA intake and limited conversion of ALA to n-3 PUFAs in humans, the DHA and EPA levels are lower in vegetarians than in omnivores. To increase the DHA and EPA levels, therefore, it is recommended that vegetarians consume ALA-rich oils to decrease the n-6/n-3 ratio to about 2 to 4 (Davis and KrisEtherton 2003). In the present study, the serum concentrations of EPA and DHA were negatively correlated with the n-6/n-3 ratio of the EN formula when DHA and EPA were not included in the formula. This finding is consistent with a previous report in which switching from an EN formula with a high $n-6 / n-3$ ratio (identical to that of the high $\mathrm{n}-6 / \mathrm{n}-3$ EN group in the present study) to one with a low $\mathrm{n}-6 / \mathrm{n}-3$ ratio (identical to that in the low $n-6 / n-3$ EN group) increased the serum EPA and DHA levels within a few months in patients receiving EN (Tanaka et al. 2004). Therefore, the biosynthesis of EPA and DHA seemed to be facilitated by EN formulas with low $n-6 / n-3$ ratios.

The EN formulas used intermediate and high n-6/n-3 EN groups differed in that the ALA concentration was increased in the former, although the LA concentrations were high in both groups. The increase in ALA concentration in intermediate n-6/n-3 EN groups did not effectively ameliorate the serum DHA and EPA levels. Conversely, the EPA and DHA levels were significantly increased in the low n-6/n-3 EN group, which received EN formulas containing ALA concentrations that were almost the same as those in the intermediate n-6/n-3 EN group but had markedly reduced LA concentrations. Therefore, it seemed that not only increasing the ALA concentration but also simultane- ously reducing the LA concentration in the EN formula effectively facilitated EPA and DHA synthesis. The conversion of ALA to EPA and DHA is suppressed by a large intake of LA (Brenna 2002); EPA synthesis was facilitated by lower levels of LA in the diet, whereas DHA synthesis was increased by higher levels of dietary ALA, resulting in an apparent dependency on the n-6/n-3 ratio (Goyens et al. 2006). However, in the present study as well as in a previous report (Tanaka et al. 2004), the effects of the reduced n-6/n-3 ratio were limited, and the serum EPA and DHA levels were still lower than those in the controls, particularly in regard to DHA.

In patients with ALA deficiency due to an EN formla containing low concentrations of ALA, supplementation with both ALA and long-chain n-3 PUFAs normalized the blood EPA and DHA status and ameliorated deficiency symptoms (Bjerve et al. 1987). Similarly, supplementation with EPA and DHA through the consumption of fish oil effectively improved the systemic EPA and DHA status in patients with phenylketonuria (Beblo et al. 2007). Among vegetarians, direct supplementation with DHA effectively increased the concentration of DHA as well as that of EPA by retroconversion of DHA in blood lipids (Geppert et al. 2005; Wu et al. 2006). Since the in vivo biosynthesis of EPA and DHA is slow and unreliable, it has been recommended that they be given as preformed nutrients in enteral nutrition (Gerster 1995). Consistent with these previous reports, we found that the serum levels of EPA and DHA reached the control ranges in the EPA + DHA-containing EN group; this observation indicates the advantage of the direct intake of preformed EPA and DHA. Therefore, although low n-6/n-3 EN formula improved the EPA and DHA levels, EN formulas containing EPA and DHA seemed to be more effective in patients requiring long-term EN.

The data pertaining to the $n-6$ series showed that the AA levels in the intermediate and high n-6/n-3 EN groups did not differ from the control values, suggesting the effective in vivo formation of AA from LA. Conversely, the serum LA and AA levels in the groups receiving EN formulas containing low LA (EPA + DHA-containing and low $\mathrm{n}-6 / \mathrm{n}-3 \mathrm{EN}$ groups) were significantly lower than those in the control group. Given that aberrant activation of the AA cascade can cause abnormal allergic responses and thrombotic reactions, these reduced AA levels may have desirable implications. Indeed, the use of a low-LA formula, identical to that in the low n-6/n-3 EN group in the present study, reduced coagulation and inflammatory activity after surgery (Aiko et al. 2005). However, with the long-term use of EN, as deficiencies in LA can precipitate clinical problems (Richardson and Sgoutas 1975), further investigation may be required to determine whether the LA intake is suitable in each patient chronically receiving a low-LA formula. In particular, in patients with limited motor activities and chronic hypothermia, the daily amount of EN can be grossly reduced to meet the low caloric requirements, which would largely restrict LA intake when a low-LA formula is used 
(Dickerson et al. 2003).

In conclusion, ALA-enriched formula with low LA concentrations may facilitate EPA and DHA production in vivo, although the EN formula containing preformed EPA and DHA was more effective. The fatty acid compositions of EN formulas strongly affected the fatty acid status of the patients on long-term EN, which indicates the need for further investigation to optimize the EN formula given to these patients.

\section{References}

Aiko, S., Yoshizumi, Y., Tsuwano, S., Shimanouchi, M., Sugiura, Y. \& Maehara, T. (2005) The effects of immediate enteral feeding with a formula containing high levels of $\omega-3$ fatty acids in patients after surgery for esophageal cancer. J. Parenter. Enteral. Nutr., 29, 141-147.

Beblo, S., Reinhardt, H., Demmelmair, H., Muntau, A.C. \& Koletzko, B. (2007) Effect of fish oil supplementation on fatty acid status, coordination, and fine motor skills in children with phenylketonuria. J. Pediatr., 150, 479-484.

Bjerve, K.S., Mostad, I.L. \& Thoresen, L. (1987) Alpha-linolenic acid deficiency in patients on long-term gastric-tube feeding: Estimation of linolenic acid and long-chain unsaturated n-3 fatty acid requirement in man. Am. J. Clin. Nutr., 45, 66-77.

Brenna, J.T. (2002) Efficiency of conversion of $\alpha$-linolenic acid to long chain n-3 fatty acids in man. Curr. Opin. Clin. Nutr. Metab. Care, 5, 127-132.

Burdge, G.C., Jones, A.E. \& Wootton SA. (2002) Eicosapentaenoic and docosapentaenoic acids are the principal products of $\alpha$-linolenic acid metabolism in young men. Br. J. Nutr., 88, 355-363.

Chida, N., Hirono, H. \& Arakawa, T. (1972) Effects of dietary folate deficiency on fatty acid composition of myelin cerebroside in growth rats. Tohoku J. Exp. Med., 108, 219-224.

Davis, B.C. \& Kris-Etherton, P.M. (2003) Achieving optimal essential fatty acid status in vegetarians: Current knowledge and practical implications. Am. J. Clin. Nutr., 78, 640S-646S.

Dickerson, R.N., Brown, R.O., Hanna, D.L. \& Williams, J.E. (2003) Energy requirements of non-ambulatory, tube-fed adult patients with cerebral palsy and chronic hypothermia. Nutrition, 19, 741-746.

Folch, J., Lees, M. \& Sloane-Stanley, G.H. (1957) A simple method for the isolation and purification of total lipids from animal tissues. J. Biol. Chem., 226, 497-509.
Geppert, J., Kraft, V., Demmelmair, H. \& Koletzko, B. (2005) Docosahexaenoic acid supplementation in vegetarians effectively increases omega-3 index: A randomized trial. Lipids, 40, 807-814.

Gerster, H. (1995) The use of n-3 PUFAs (fish oil) in enteral nutrition. Int. J. Vitam. Nutr. Res., 65, 3-20.

Goyens, P.L., Spilker, M.E., Zock, P.L., Katan, M.B. \& Mensink, R.P. (2006) Conversion of $\alpha$-linolenic acid in humans is influenced by the absolute amounts of $\alpha$-linolenic acid and linoleic acid in the diet and not by their ratio. Am. J. Clin. Nutr., 84, 44-53.

Holman, R.T., Johnson, S.B. \& Hatch, T.F. (1982) A case of human linolenic acid deficiency involving neurological abnormalities. Am. J. Clin. Nutr., 35, 617-623.

Innis, S.M. (2007) Dietary (n-3) fatty acids and brain development. J. Nutr., 137, 855-859.

Kinsella, J.E., Lokesh, B. \& Stone, R.A. (1990) Dietary n-3 polyunsaturated fatty acids and amelioration of cardiovascular disease: Possible mechanisms. Am. J. Clin. Nutr., 52, 1-28.

Kumode, M. (2003) Management of nutrition in children and adults with severe motor and intellectual disabilities. No To Hattatsu (Tokyo), 35, 206-210.

Munakata, M., Kobayashi, K., Niisato-Nezu, J., Tanaka, S., Kakisaka, Y., Ebihara, T., Ebihara, S., Haginoya, K., Tsuchiya, S. \& Onuma A. (2008) Olfactory stimulation using black pepper oil facilitates oral feeding in pediatric patients receiving long-term enteral nutrition. Tohoku J. Exp. Med., 214, 327-332.

R Development Core Team. (2005) R: A language and environment for statistical computing. $R$ Foundation for Statistical Computing, Vienna, Austria.

Richardson, T.J. \& Sgoutas, D. (1975) Essential fatty acid deficiency in four adult patients during total parenteral nutrition. Am. J. Clin. Nutr., 28, 258-263.

Sugano, M. (1996) Characteristics of fats in Japanese diets and current recommendations. Lipids, 31, S283-S286.

Tanaka, Y., Mizote, H., Inada, H., Motohiro, T., Kobayashi, H., Fukahori, S., So, H., Otani, M., Nakamizo, H., Asagiri, K., Akiyoshi, K., Tsuru, T. \& Hikida, S. (2004) Efficacy of n-3 polyunsaturated fatty acid enriched enteral nutrient solution in relieving oxidative stress in patients with severe psychophysiologic disorders. Kurume Med. J., 51, 83-90.

Wu, W.H., Lu, S.C., Wang, T.F., Jou, H.J. \& Wang, T.A. (2006) Effects of docosahexaenoic acid supplementation on blood lipids, estrogen metabolism, and in vivo oxidative stress in postmenopausal vegetarian women. Eur. J. Clin. Nutr., 60, 386-392. 